\title{
A novel one-pot synthesis of some new interesting pyrrole derivatives
}

Javad Azizian,* Ali R. Karimi, Zahra Kazemizadeh, Ali A. Mohammadi, Mohammad R.

Mohammadizadeh

Department of Chemistry, Faculty of Science, Shahid Beheshti University, P. O. Box 19395-

4716, Tehran, Iran,

E-mail: j-azizian@cc.sbu.ac.ir

\section{SUPPORTING INFORMATION}

Detailed list of CONTENTS of the supporting information

\begin{tabular}{|c|c|c|c|}
\hline No & List of contents & Supporting info. No. & page \\
\hline 01 & Title, author's name, address and Table & - & S1 \\
\hline 02 & Experimental Section & - & S2 \\
\hline 03 & $\begin{array}{l}\text { General procedure for preparation of } \mathbf{3 a - c} \\
\text { and } \mathbf{5 a - 5 d : ~}\end{array}$ & - & S2 \\
\hline 04 & Spectral data for Products 3a-c and 5a-d & - & S2 \\
\hline 05 & Mass of 3a & S01 & S6 \\
\hline 06 & Mass of 3c & S02 & S7 \\
\hline 07 & Mass of 5c & S03 & S8 \\
\hline 08 & Mass of 5d & S04 & S9 \\
\hline 09 & ${ }^{1} \mathrm{H}$ NMR of 3a & S05 & S10 \\
\hline 10 & ${ }^{13} \mathrm{C}$ NMR of $3 a$ & S06 & S11 \\
\hline 11 & ${ }^{13} \mathrm{C}$ NMR of $3 a($ expanded) & S07 & S12 \\
\hline 12 & ${ }^{1} \mathrm{H}$ NMR of $3 \mathrm{c}$ & S08 & S13 \\
\hline 13 & ${ }^{13} \mathrm{C}$ NMR of $3 \mathrm{c}$ & S09 & S14 \\
\hline 14 & ${ }^{1} \mathrm{H}$ NMR of $5 c$ & S10 & S15 \\
\hline 15 & ${ }^{13} \mathrm{C}$ NMR of $5 c$ & S11 & S16 \\
\hline 16 & ${ }^{1}$ H NMR of $5 d$ & S12 & $\mathrm{S} 17$ \\
\hline 17 & ${ }^{13} \mathrm{C}$ NMR of $5 d$ & S13 & S18 \\
\hline
\end{tabular}




\section{Experimental Section:}

Melting points were measured on the Electrothermal 9100 apparatus and are uncorrected. IR spectra were measured on a Shimadzu IR-470 Spectrophotometer. ${ }^{1} \mathrm{H}$ NMR and ${ }^{13} \mathrm{C}$ NMR spectra were determined on a Bruker 500 and 300 DRX AVNCE instrument at 500 or 300 and 125 or $75 \mathrm{MHz}$, respectively. Elemental analyses were performed using a Heracus CHN-ORapid analyzer. Microwave irradiation were carried out in a National oven, model 5250, at 2450 $\mathrm{MHz}$.

\section{General procedure for preparation of 3a-c and 5a-5d:}

Montmorillonite K10 (0.5 g) was placed in a mortar followed by addition of $11 \mathrm{H}$-indeno[1,2b]quinoxalin-11-ones 1a-c or isatin derivatives 4a-d (1 mmol) and 4-hydroxy proline $2(0.131 \mathrm{~g}$, $1 \mathrm{mmol}$ ) to which was added five drop of DMSO as a wet solvent. These materials were then mixed using a pestle for ca. $5 \mathrm{~min}$. The homogenized mixture was placed in a pyrex test tube and then loaded into the microwave oven and irradiated for 2 min with a power of $900 \mathrm{w}$. The contents were cooled to room temperature and mixed thoroughly with $10 \mathrm{~mL}$ acetone. The solid inorganic material was filtered off. After separation of montmorillonite K10, water added to the mixture and the separated solid was filtered off and dried under high vaccum and recrystallised in ethanol to give a pure crystalline products.

\section{Spectral data for Products 3a-c and 5a-d:}

11-(1H-pyrrol-1-yl)-11H-indeno[1,2-b]quinoxaline 3a: mp: $180-181{ }^{\circ} \mathrm{C}, \mathrm{IR}(\mathrm{KBr})\left(v_{\max }, \mathrm{cm}^{-1}\right)$ : 3095, 2920, 1478; ${ }^{1} \mathrm{H}$ NMR $\left(\mathrm{CDCl}_{3}, 500 \mathrm{MHz}\right) \delta_{\mathrm{H}}: 6.21(1 \mathrm{H}, \mathrm{s}, \mathrm{CH}), 6.23(2 \mathrm{H}, \mathrm{t}, \mathrm{J}=2.10 \mathrm{~Hz}$, $\left.\mathrm{CH}_{\text {pyrrole }}\right), 6.74\left(2 \mathrm{H}, \mathrm{t}, J=2.09 \mathrm{~Hz}, \mathrm{CH}_{\text {pyrrole }}\right), 7.58-8.29$ (8H, m, Arom); ${ }^{13} \mathrm{C} \mathrm{NMR}\left(\mathrm{CDCl}_{3}, 75\right.$ MHz) $\delta_{\mathrm{C}}: 61.63(\mathrm{CH}), 109.30,120.40\left(2 \mathrm{CH}_{\text {pyrrole }}\right), 122.78,125.99,128.99,129.36,129.81$, $130.34,130.38,132.19,137.19,141.67,142.59,143.80,153.17,158.16$ (arom). MS (m/z, \%): 
$283\left(\mathrm{M}^{+}, 100\right), 217$ (85), 190 (60), 141 (15), 114 (15), 89 (45), 63 (16), 39 (50). Anal. Calcd for $\mathrm{C}_{19} \mathrm{H}_{13} \mathrm{~N}_{3}: \mathrm{C}, 80.54 ; \mathrm{H}, 4.62 ; \mathrm{N}, 14.83$. Found: $\mathrm{C}, 80.58 ; \mathrm{H}, 4.63 ; \mathrm{N}, 14.81$.

8-methyl-11-(1H-pyrrol-1-yl)-11H-indeno[1,2-b]quinoxaline 3b: mp: 192-193 ${ }^{\circ} \mathrm{C}$, IR (KBr) $\left(v_{\max }, \mathrm{cm}^{-1}\right): 3098,2918,1483 ;{ }^{1} \mathrm{H}$ NMR $\left(\mathrm{CDCl}_{3}, 300 \mathrm{MHz}\right) \delta_{\mathrm{H}}: 2.66\left(3 \mathrm{H}, \mathrm{s}, \mathrm{CH}_{3}\right), 6.25(1 \mathrm{H}, \mathrm{s}$, $\mathrm{CH}), 6.27$ (2H, bs, $\left.\mathrm{CH}_{\text {pyrrole }}\right), 6.74\left(2 \mathrm{H}, \mathrm{t}, \mathrm{J}=1.80 \mathrm{~Hz}, \mathrm{CH}_{\text {pyrrole }}\right), 7.62-8.23$ (7H, m, Arom); ${ }^{13} \mathrm{C}$ NMR $\left(\mathrm{CDCl}_{3}, 75 \mathrm{MHz}\right) \delta_{\mathrm{C}}: 22.08\left(\mathrm{CH}_{3}\right), 62.13(\mathrm{CH}), 109.55,120.44\left(2 \mathrm{CH}_{\text {pyrrole }}\right), 122.58$, $124.70,128.69,130.45,131.13,132.55,136.70,141.14,141.38,142.88,143.88,148.42,153.17$, 158.16 (arom). MS (m/z, \%): $297\left(\mathrm{M}^{+}, 30\right), 246$ (100), 231 (55), 218 (60), 190 (15), 130 (15), 89 (30). Anal. Calcd for $\mathrm{C}_{20} \mathrm{H}_{15} \mathrm{~N}_{3}$ : C, 80.78; H, 5.08; N, 14.13. Found: C, 80.77; H, 5.05; N, 14.11 . 7,8-dimethyl-11-(1H-pyrrol-1-yl)-11H-indeno[1,2-b]quinoxaline 3c: mp: $231-232{ }^{\circ} \mathrm{C}$, IR (KBr) $\left(v_{\max }, \mathrm{cm}^{-1}\right): 3120,2915,1486,1329 ;{ }^{1} \mathrm{H}$ NMR $\left(\mathrm{CDCl}_{3}, 500 \mathrm{MHz}\right) \delta_{\mathrm{H}}: 2.44$ and $2.47(6 \mathrm{H}, 2 \mathrm{~s}$, $\left.2 \mathrm{CH}_{3}\right), 6.12(1 \mathrm{H}, \mathrm{s}, \mathrm{CH}), 6.23\left(2 \mathrm{H}, \mathrm{t}, J=1.26 \mathrm{~Hz}, \mathrm{CH}_{\text {pyrrole }}\right), 6.73\left(2 \mathrm{H}, \mathrm{t}, J=1.33 \mathrm{~Hz}, \mathrm{CH}_{\text {pyrrole }}\right)$, 7.50-8.19 (6H, m, Arom); ${ }^{13} \mathrm{C} \mathrm{NMR}\left(\mathrm{CDCl}_{3}, 125 \mathrm{MHz}\right) \delta_{\mathrm{C}}: 20.23$ and $20.35\left(2 \mathrm{CH}_{3}\right), 61.66(\mathrm{CH})$, $109.17,120.37\left(2 \mathrm{CH}_{\text {pyrrole }}\right), 122.29,125.83,128.33,129.00,130.16,131.54,137.67,139.72$, 140.50, 140.65, 141.60, 143.56, 152.37, 157.14 (arom). MS (m/z, \%): $311\left(\mathrm{M}^{+}, 100\right), 245(90)$, 229 (30), 103 (15), 77 (15), 39 (45). Anal. Calcd for $\mathrm{C}_{21} \mathrm{H}_{17} \mathrm{~N}_{3}: \mathrm{C}, 81.00 ; \mathrm{H}, 5.50 ; \mathrm{N}, 13.49$. Found: C, 79.88; H, 5.55; N, 13.51.

3-(1H-pyrrol-1-yl)indolin-2-one 5a: $\mathrm{mp}: 143-144{ }^{\circ} \mathrm{C}$, IR (KBr) $\left(v_{\max }, \mathrm{cm}^{-1}\right): 3295,3190,1711$, 1614, 1482; ${ }^{1} \mathrm{H}$ NMR $\left(\mathrm{CDCl}_{3}, 300 \mathrm{MHz}\right) \delta_{\mathrm{H}}: 6.51(1 \mathrm{H}, \mathrm{s}, \mathrm{CH}), 6.25\left(2 \mathrm{H}, \mathrm{bs}, \mathrm{CH}_{\text {pyrrole }}\right), 6.70(2 \mathrm{H}$, bs, $\left.\mathrm{CH}_{\text {pyrrole }}\right), 6.85-7.34$ (4H, m, Arom), $8.87(1 \mathrm{H}, \mathrm{bs}, \mathrm{NH}) ;{ }^{13} \mathrm{C} \mathrm{NMR}\left(\mathrm{CDCl}_{3}, 75 \mathrm{MHz}\right) \delta_{\mathrm{C}}$ : $60.85(\mathrm{CH}), 109.54,110.76\left(2 \mathrm{CH}_{\text {pyrrole }}\right), 120.33,123.30,125.10,125.53,130.21,141.31$ (arom), $175.16(\mathrm{C}=\mathrm{O}) . \mathrm{MS}(\mathrm{m} / \mathrm{z}, \%): 198\left(\mathrm{M}^{+}, 100\right), 169$ (85), 132 (90), 104 (10), 39 (10). Anal. Calcd for $\mathrm{C}_{12} \mathrm{H}_{10} \mathrm{~N}_{2} \mathrm{O}$ : C, 72.71; H, 5.08; N, 14.13; O, 8.07. Found: C, 72.73; H, 5.09; N, 14.12. 
1-methyl-3-(1H-pyrrol-1-yl)indolin-2-one 5b: mp: 133-134 ${ }^{\circ} \mathrm{C}$, IR $(\mathrm{KBr})\left(v_{\max }, \mathrm{cm}^{-1}\right): 1712$, 1606, 1486; ${ }^{1} \mathrm{H}$ NMR $\left(\mathrm{CDCl}_{3}, 300 \mathrm{MHz}\right) \delta_{\mathrm{H}}: 3.23\left(3 \mathrm{H}, \mathrm{s}, \mathrm{CH}_{3}\right), 5.48(1 \mathrm{H}, \mathrm{s}, \mathrm{CH}), 6.22(2 \mathrm{H}, \mathrm{bs}$, $\left.\mathrm{CH}_{\text {pyrrole }}\right), 6.68\left(2 \mathrm{H}, \mathrm{bs}, \mathrm{CH}_{\text {pyrrole }}\right), 6.89-7.42\left(4 \mathrm{H}, \mathrm{m}\right.$, Arom); ${ }^{13} \mathrm{C} \mathrm{NMR}\left(\mathrm{CDCl}_{3}, 75 \mathrm{MHz}\right) \delta_{\mathrm{C}}$ : $26.42\left(\mathrm{CH}_{3}\right), 60.29(\mathrm{CH}), 108.65,109.36\left(2 \mathrm{CH}_{\text {pyrrole }}\right), 120.21,123.23,124.53,125.31,130.20$, 144.17 (arom), $172.45(\mathrm{C}=\mathrm{O})$. MS (m/z, \%): $198\left(\mathrm{M}^{+}, 100\right), 169$ (85), 132 (90), 104 (10), 39 (10). Anal. Calcd for $\mathrm{C}_{13} \mathrm{H}_{12} \mathrm{~N}_{2} \mathrm{O}$ : C, 73.56; H, 5.70; N, 13.20; O, 7.54. Found: C, 73.54; H, 5.72; N, 13.18.

1-benzyl-3-(1H-pyrrol-1-yl)indolin-2-one 5c: mp: 126-127 ${ }^{\circ} \mathrm{C}, \mathrm{IR}(\mathrm{KBr})\left(v_{\max }, \mathrm{cm}^{-1}\right)$ : 1713, 1609, 1485; ${ }^{1} \mathrm{H}$ NMR $\left(\mathrm{CDCl}_{3}, 300 \mathrm{MHz}\right) \delta_{\mathrm{H}}: 4.93\left(2 \mathrm{H}, \mathrm{d},{ }^{2} \mathrm{~J}=7.00 \mathrm{~Hz}, \mathrm{CH}_{2}\right), 5.58(1 \mathrm{H}, \mathrm{s}, \mathrm{CH})$, $6.25\left(2 \mathrm{H}, \mathrm{t}, J=1.84 \mathrm{~Hz}, \mathrm{CH}_{\text {pyrrole }}\right), 6.71\left(2 \mathrm{H}, \mathrm{t}, J=1.86 \mathrm{~Hz}, \mathrm{CH}_{\text {pyrrole }}\right), 6.79-7.31$ (9H, m, Arom); ${ }^{13} \mathrm{C}$ NMR $\left(\mathrm{CDCl}_{3}, 75 \mathrm{MHz}\right) \delta_{\mathrm{C}}: 44.12\left(\mathrm{CH}_{2}\right), 60.40(\mathrm{CH}), 109.56,109.78\left(2 \mathrm{CH}_{\text {pyrrole }}\right), 120.30$, $123.34,124.66,125.45,127.41,127.91,128.95,130.16,135.35,143.31(\operatorname{arom}), 172.68(\mathrm{C}=\mathrm{O})$. MS (m/z, \%): $288\left(\mathrm{M}^{+}, 65\right), 222$ (100), 197 (10), 91 (90), 65 (10). Anal. Calcd for $\mathrm{C}_{13} \mathrm{H}_{12} \mathrm{~N}_{2} \mathrm{O}: \mathrm{C}$, 73.56; H, 5.70; N, 13.20; O, 7.54. Found: C, 73.53; H, 5.77; N, 13.30 .

5-methyl-3-(1H-pyrrol-1-yl)indolin-2-one 5d: mp: 163-164 ${ }^{\circ} \mathrm{C}, \mathrm{IR}(\mathrm{KBr})\left(v_{\max }, \mathrm{cm}^{-1}\right)$ : 3200, 1708, 1619, 1487; ${ }^{1} \mathrm{H}$ NMR $\left(\mathrm{CDCl}_{3}, 300 \mathrm{MHz}\right) \delta_{\mathrm{H}}: 4.93\left(3 \mathrm{H}, \mathrm{s}, \mathrm{CH}_{3}\right), 5.58(1 \mathrm{H}, \mathrm{s}, \mathrm{CH}), 6.25$ (2H, t, J=1.96 Hz, $\left.\mathrm{CH}_{\text {pyrrole }}\right), 6.71\left(2 \mathrm{H}, \mathrm{t}, J=2.01 \mathrm{~Hz}, \mathrm{CH}_{\text {pyrrole }}\right), 6.79-7.31$ (4H, m, Arom), 9.09 $(1 \mathrm{H}$, bs, $\mathrm{NH}) ;{ }^{13} \mathrm{C} \mathrm{NMR}\left(\mathrm{CDCl}_{3}, 75 \mathrm{MHz}\right) \delta_{\mathrm{C}}: 44.12\left(\mathrm{CH}_{3}\right), 60.40(\mathrm{CH}), 109.54,110.76$ $\left(2 \mathrm{CH}_{\text {pyrrole }}\right), 120.33,123.30,125.10,125.53,130.21,141.31$ (arom), $175.16(\mathrm{C}=\mathrm{O}) . \mathrm{MS}(\mathrm{m} / \mathrm{z}$, \%): $288\left(\mathrm{M}^{+}, 65\right), 222$ (100), $197(10), 91(90), 65(10)$. Anal. Calcd for $\mathrm{C}_{19} \mathrm{H}_{16} \mathrm{~N}_{2} \mathrm{O}: \mathrm{C}, 79.14 ; \mathrm{H}$, 5.59; N, 9.72; O, 5.55. Found: C, 79.15; H, 5.60; N, 9.77.

5-bromo3-(1H-pyrrol-1-yl)indolin-2-one 5e: mp: $171-172{ }^{\circ} \mathrm{C}$, IR $(\mathrm{KBr})\left(v_{\max }, \mathrm{cm}^{-1}\right): 3240,1714$, 1610, 1487; ${ }^{1} \mathrm{H}$ NMR $\left(\mathrm{CDCl}_{3}, 300 \mathrm{MHz}\right) \delta_{\mathrm{H}}: 5.50(1 \mathrm{H}, \mathrm{s}, \mathrm{CH}), 6.26\left(2 \mathrm{H}, \mathrm{s}, \mathrm{CH}_{\text {pyrrole }}\right), 6.67(2 \mathrm{H}$, 
s, $\left.\mathrm{CH}_{\text {pyrrole }}\right), 6.78-7.45(3 \mathrm{H}, \mathrm{m}, \mathrm{Arom}), 9.07(1 \mathrm{H}, \mathrm{bs}, \mathrm{NH}) ;{ }^{13} \mathrm{C} \mathrm{NMR}\left(\mathrm{CDCl}_{3}, 75 \mathrm{MHz}\right) \delta_{\mathrm{C}}: 60.75$ (CH), 109.90, $112.43\left(2 \mathrm{CH}_{\text {pyrrole }}\right), 115.95,120.33,127.12,128.49,133.16,140.26$ (arom), $174.96(\mathrm{C}=\mathrm{O})$. MS (m/z, \%): $278\left(\mathrm{M}^{+}+2,80\right), 276\left(\mathrm{M}^{+}, 80\right), 249(15), 247$ (15), 212 (95), 210 (100), 168 (20), 103 (30), 67 (85), 39 (50). Anal. Calcd for $\mathrm{C}_{12} \mathrm{H}_{9} \mathrm{BrN}_{2} \mathrm{O}: \mathrm{C}, 52.01$; H, 3.27; $\mathrm{Br}$, 28.83; N, 10.11; O, 5.77. Found: C, 52.08; H, 3.28; Br, 28.81; N, 10.12. 


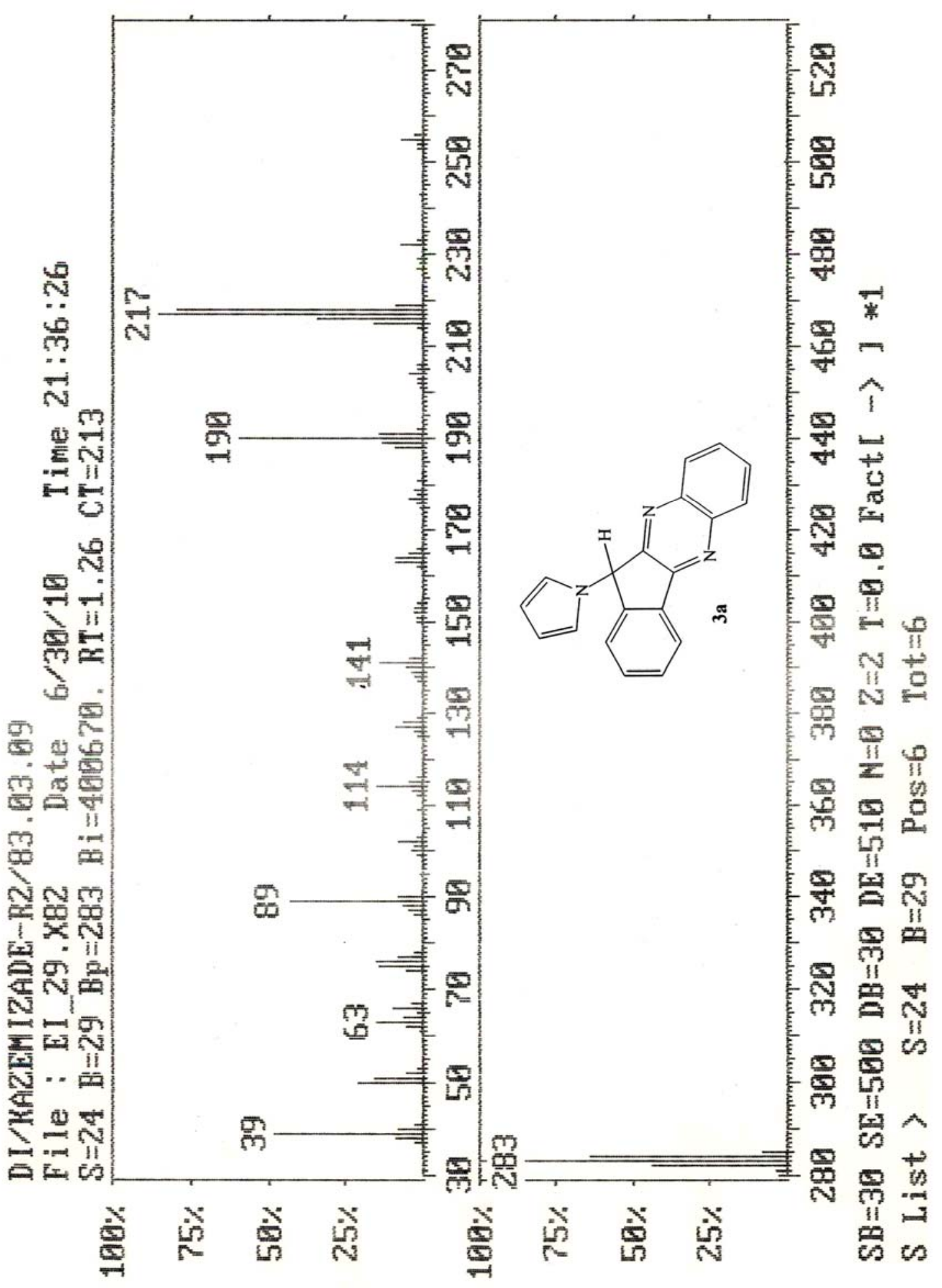




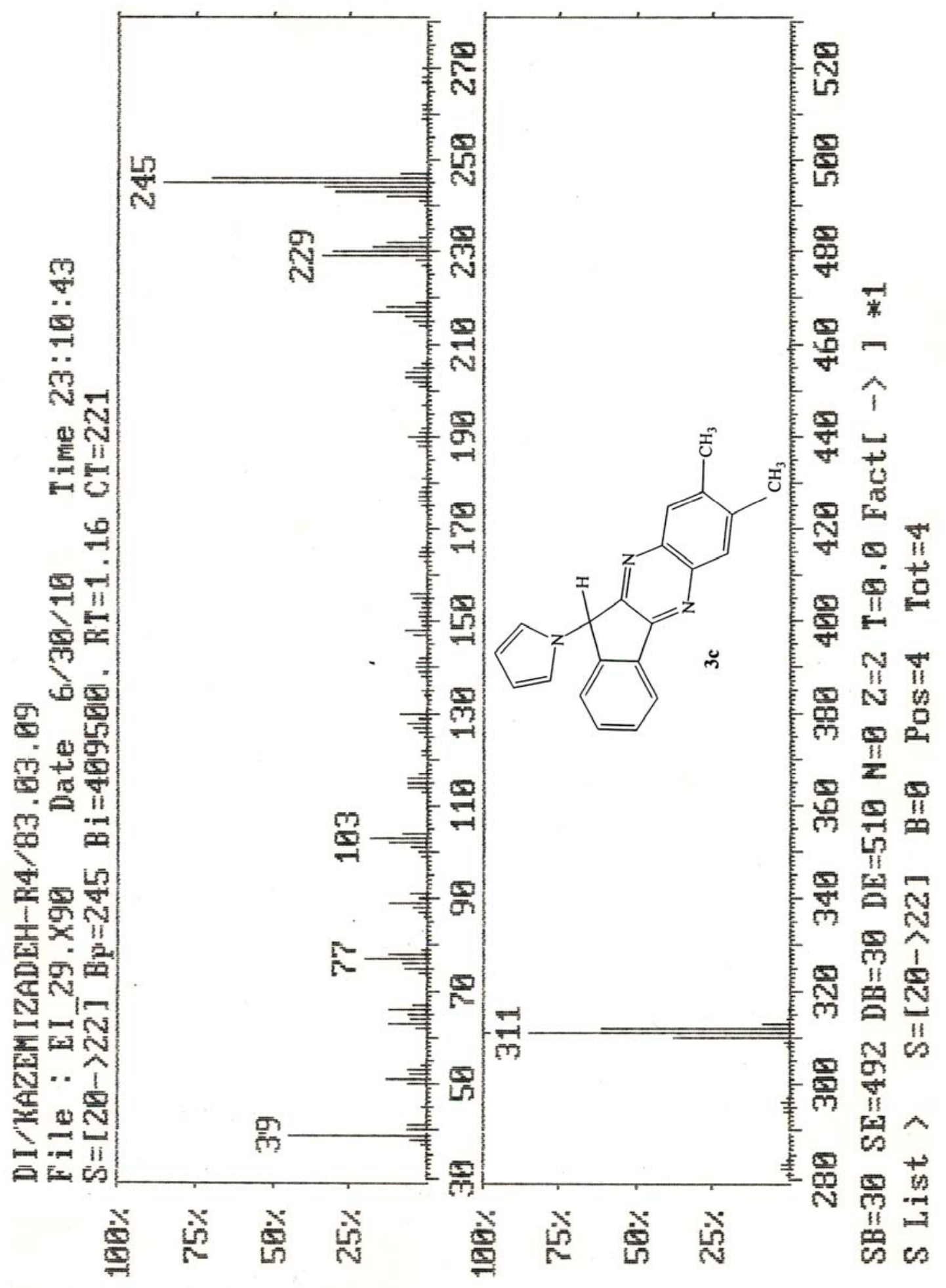




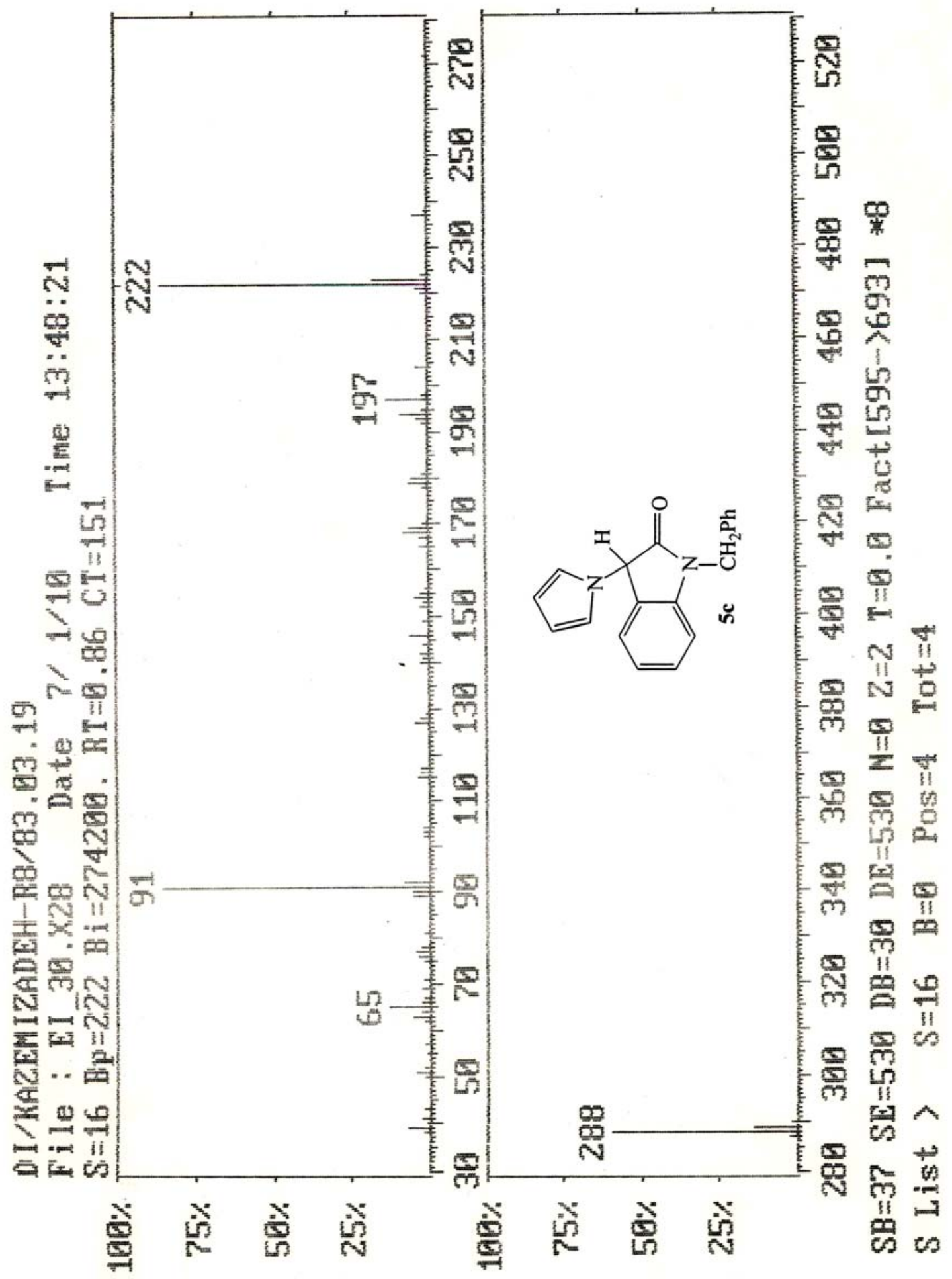




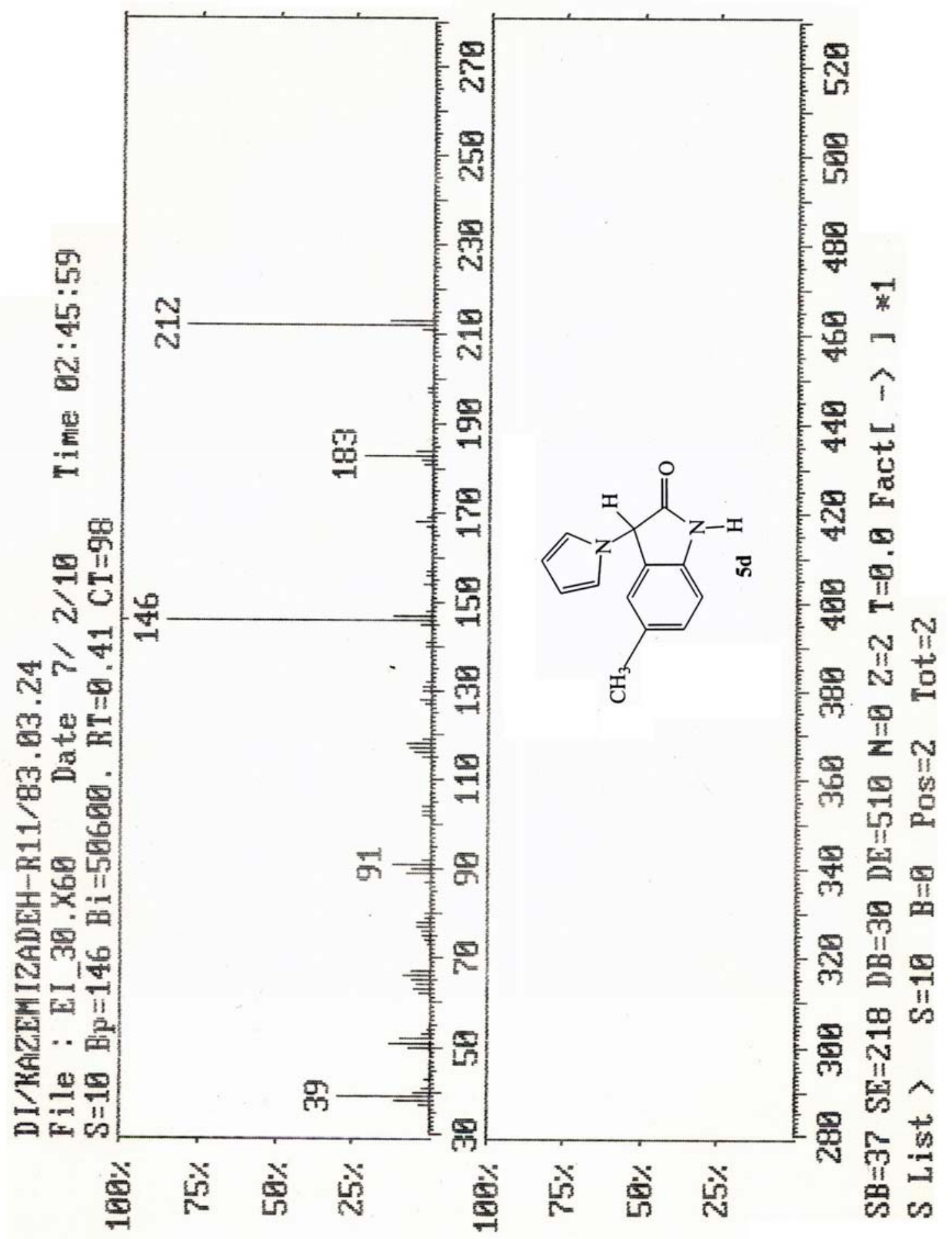




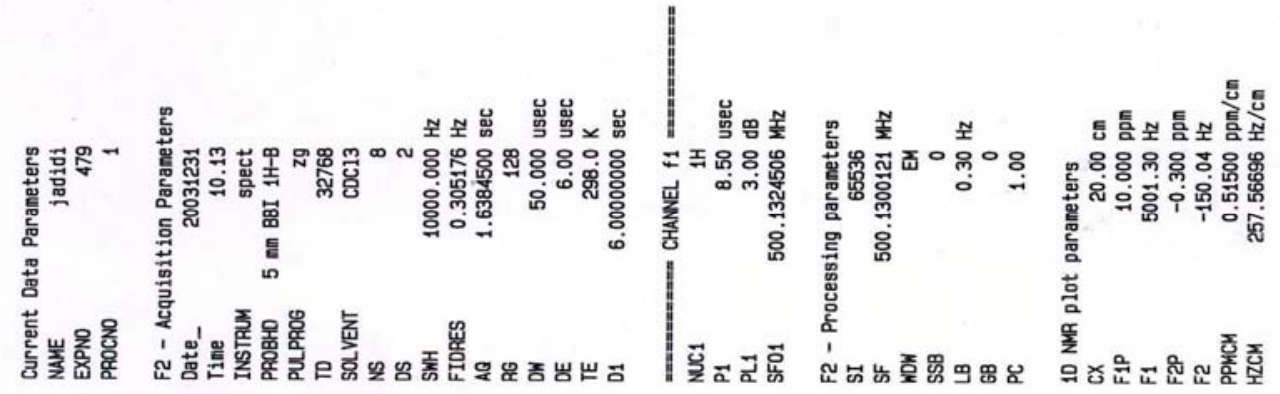

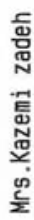
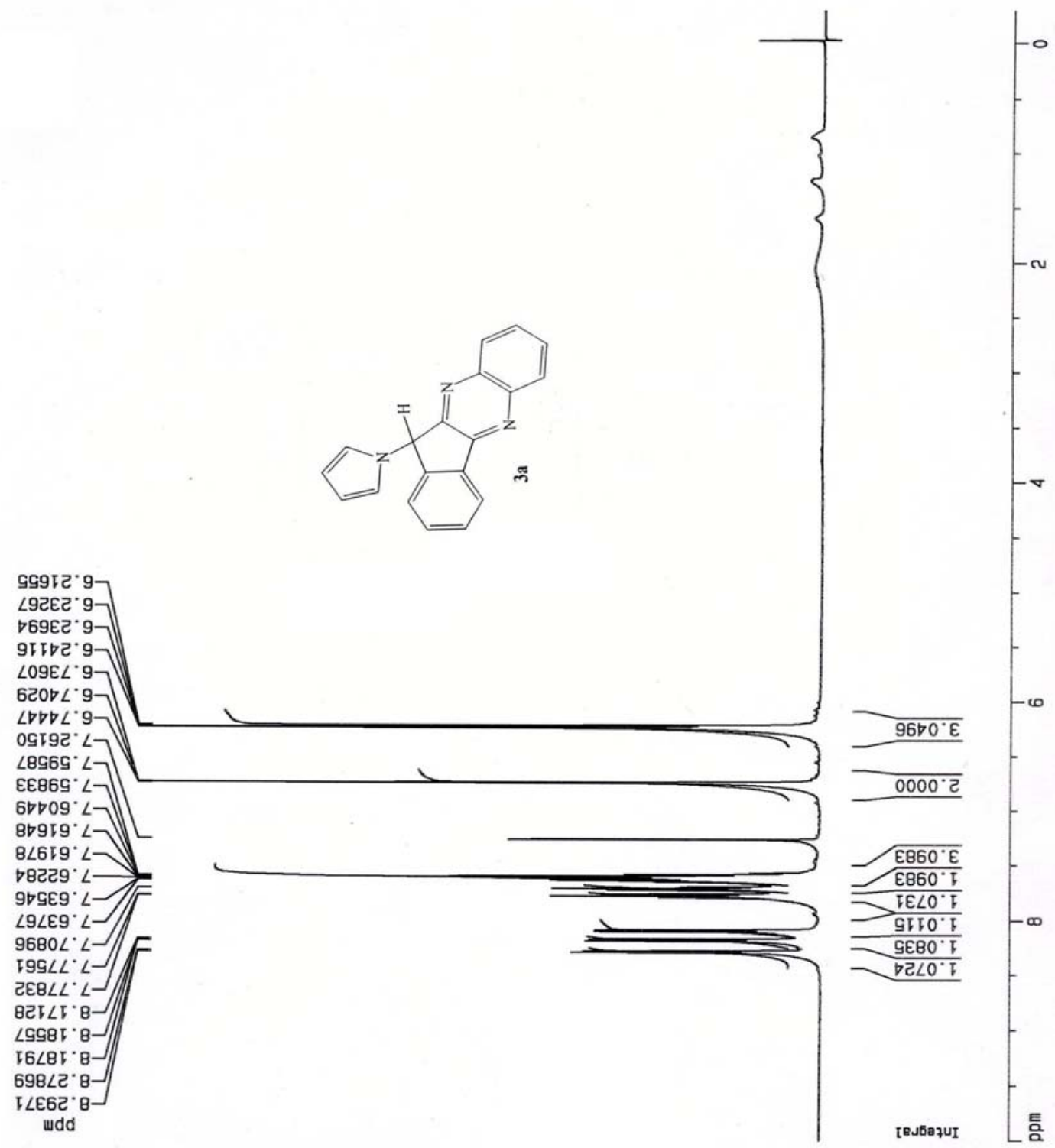

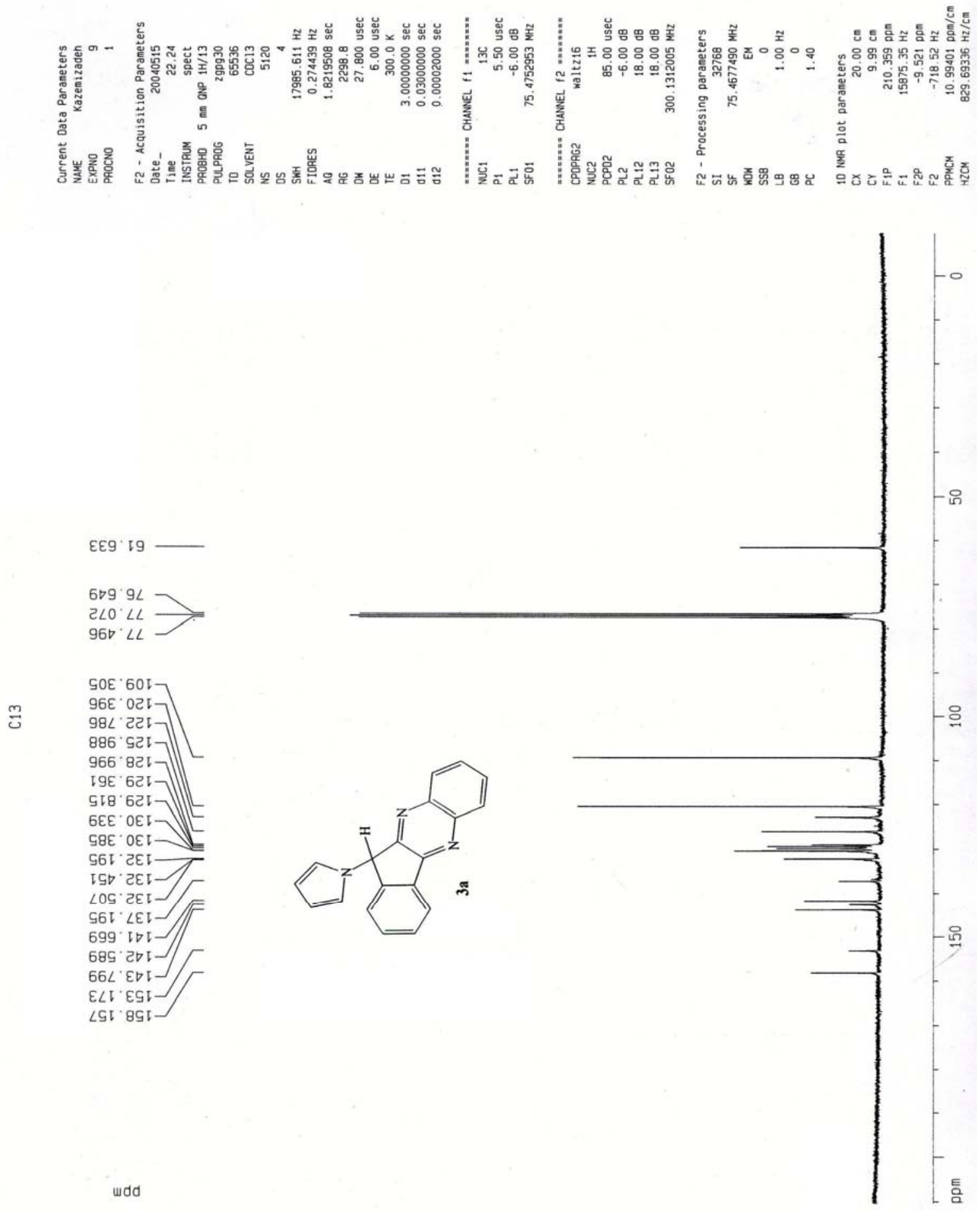

wdd 


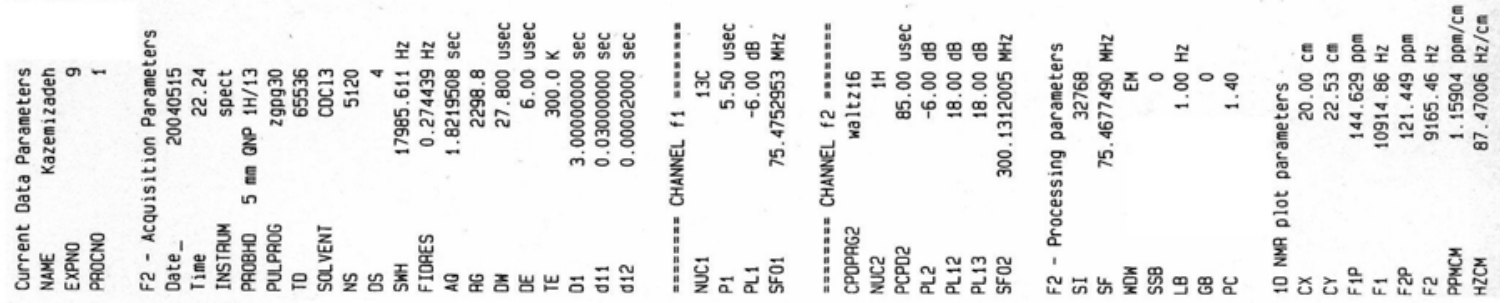

$\varepsilon 8 L^{\prime}$ टટ -

986 . 921

8GE 62 โ

$\checkmark$ ऽ 62 โ

$\angle E E^{\circ} 0 \varepsilon \Gamma$

$\varepsilon 8 \varepsilon^{\circ} 0 \varepsilon[-$

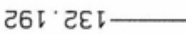

$\stackrel{9}{5}$

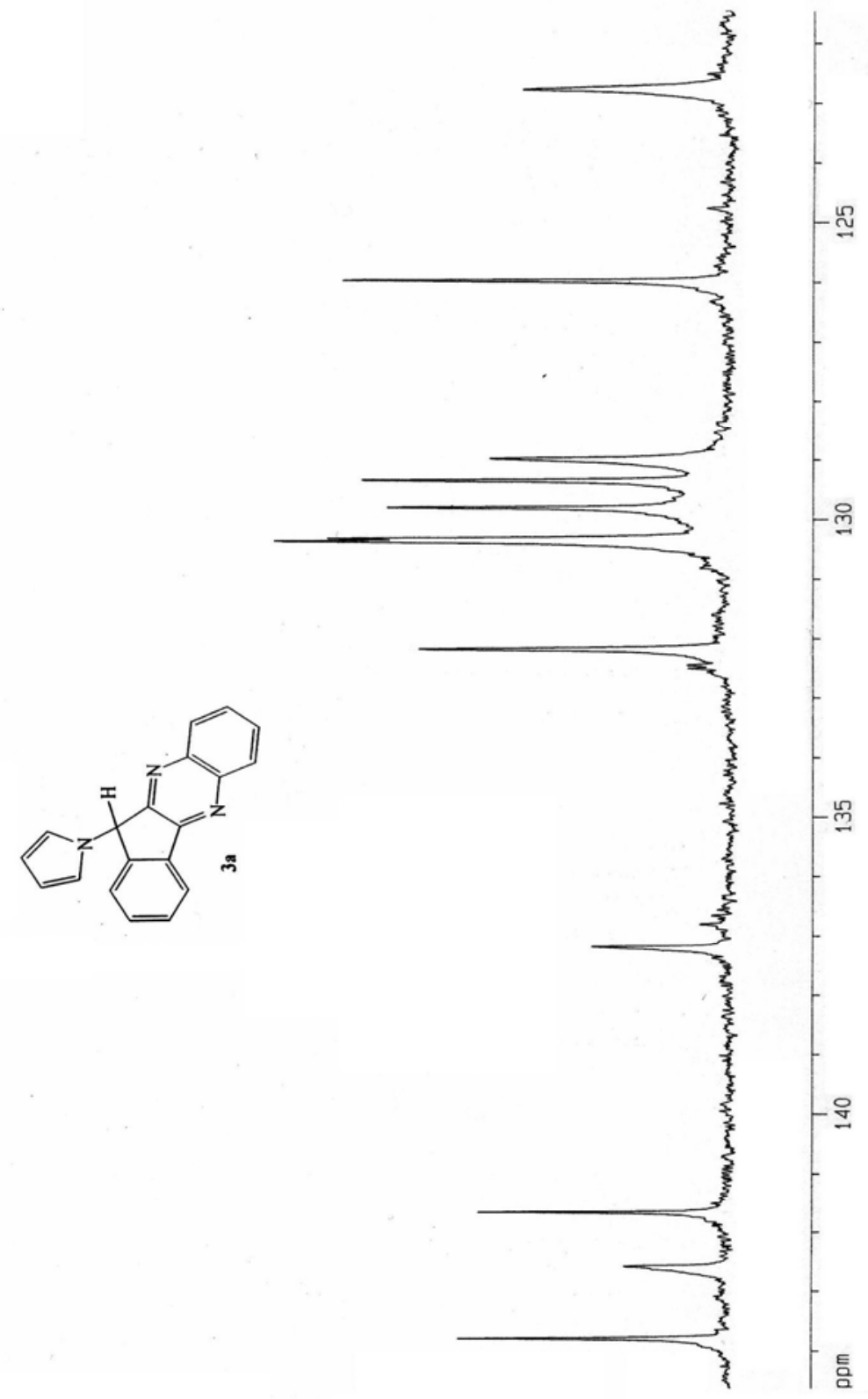

$26 \tau \cdot \angle E I-$ 


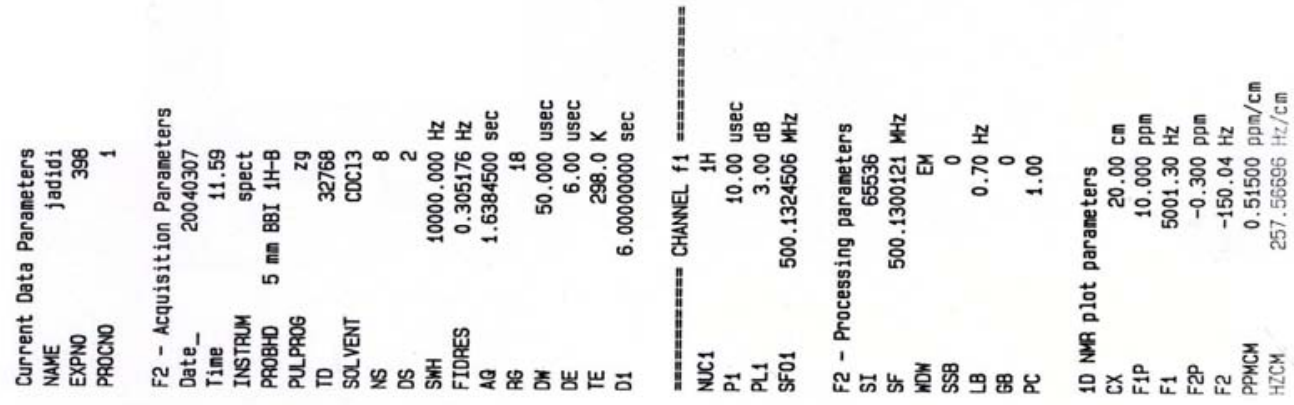

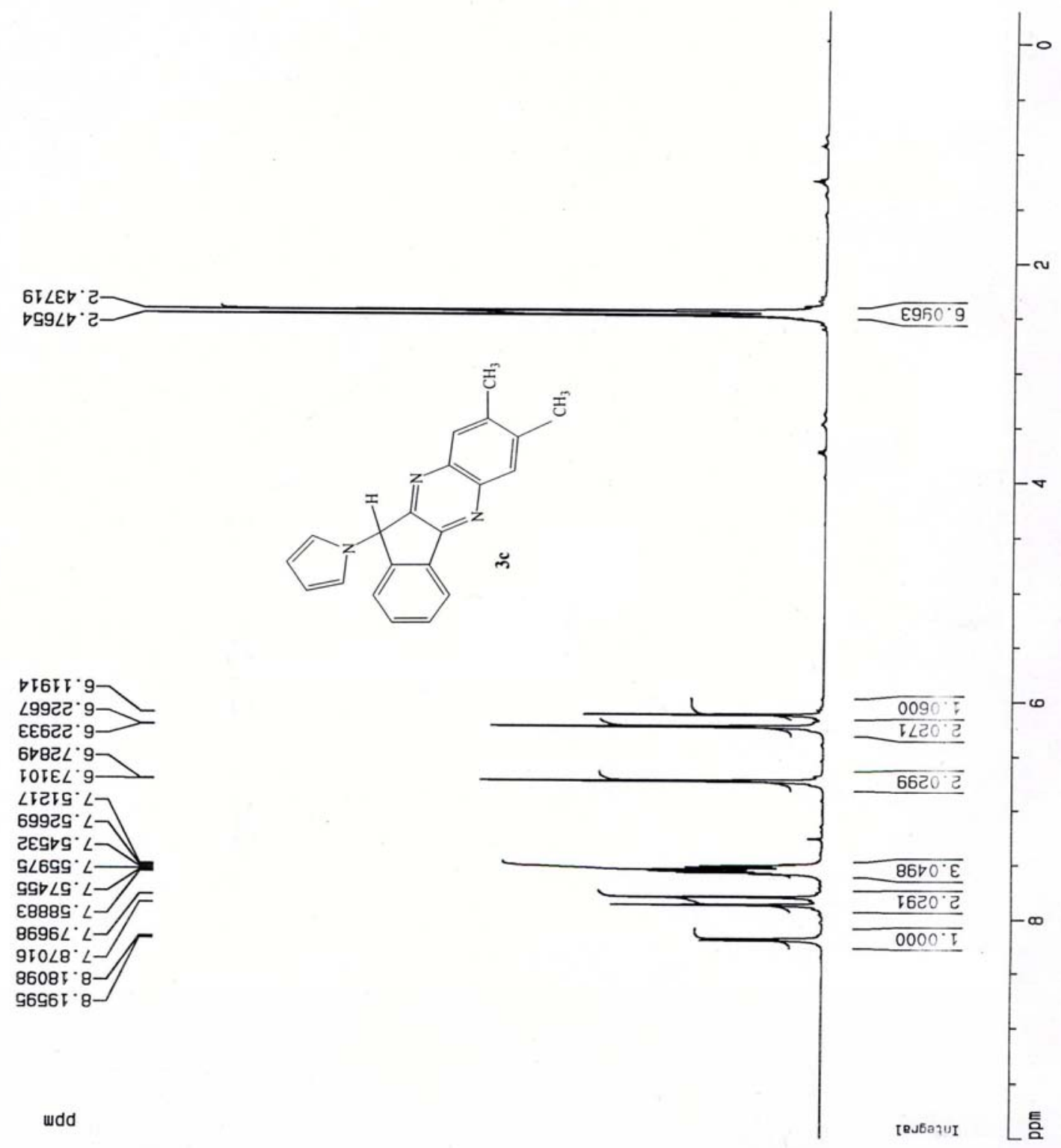




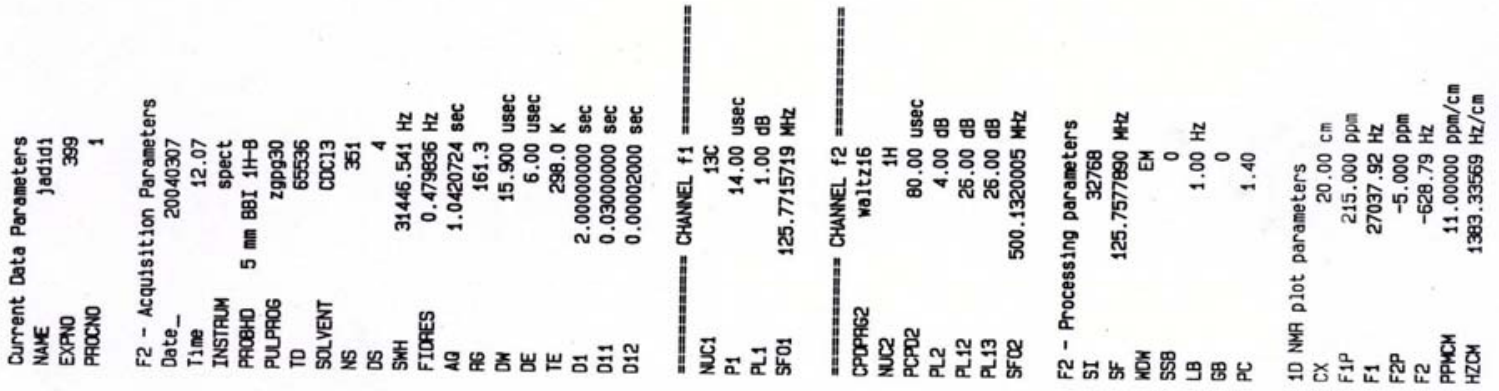

EEC 02 ESE 02

เ99* โ9

$098^{\circ} 9 L$

$\angle 9 E^{\circ} \angle L$

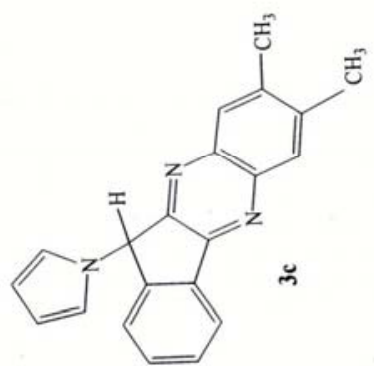

घ

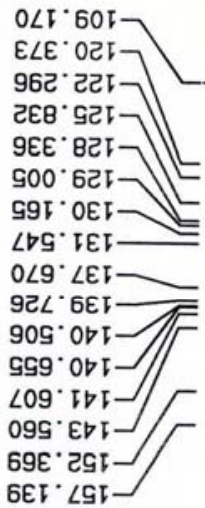

wdd

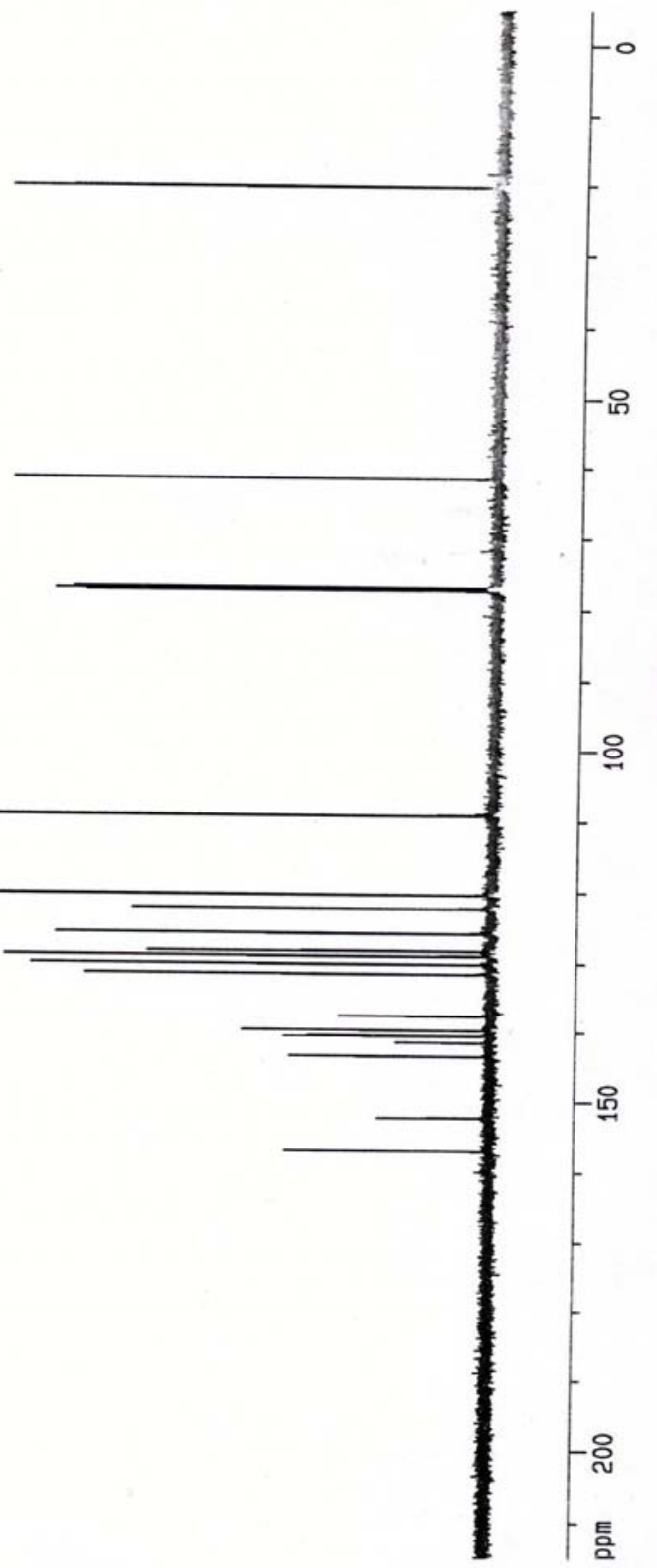




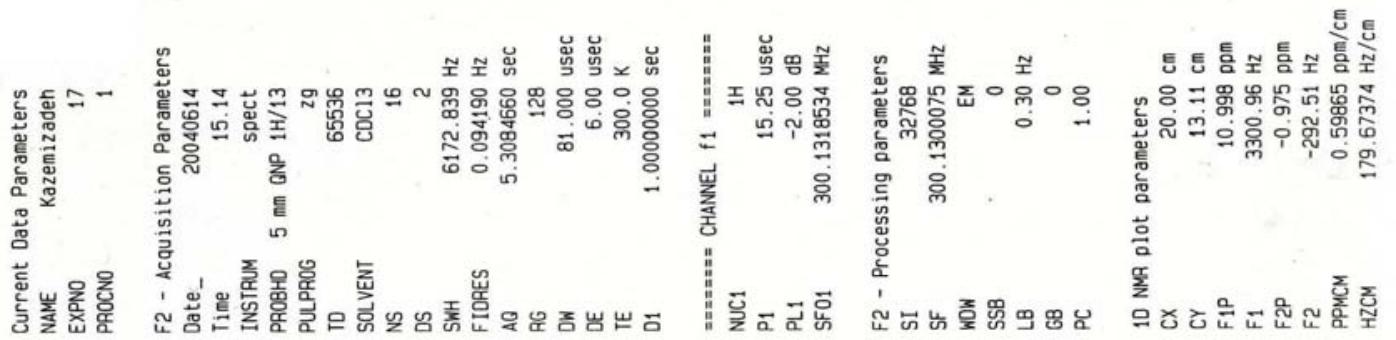

0290

5810.0

$6200^{\circ} 0$

$0820^{\circ} 0$

$\checkmark 690^{\circ} 0$<smiles>[Z]O[Z]1C(=O)C([3H])([Te]2C=CC=C2)c2ccccc21</smiles>

टจ98 $\triangleright$

โ9เ6. $\nabla$

또

SOE6 $\nabla$

$\triangle 286^{\circ} \nabla$

LOBS' 9

$66 \nabla 2.97$

0952 9

દट9ટ' 9

ट90L' 9

EटाL' 9

S8IL' 9

$8 \angle 8 \angle ' 9$

LEL8. 9

BIVO L

$\checkmark 990^{\circ} \mathrm{L}$

9160 <

S口G ' $\angle$

6982 ' $L$

EE $\left[\varepsilon^{\circ}<-\right.$

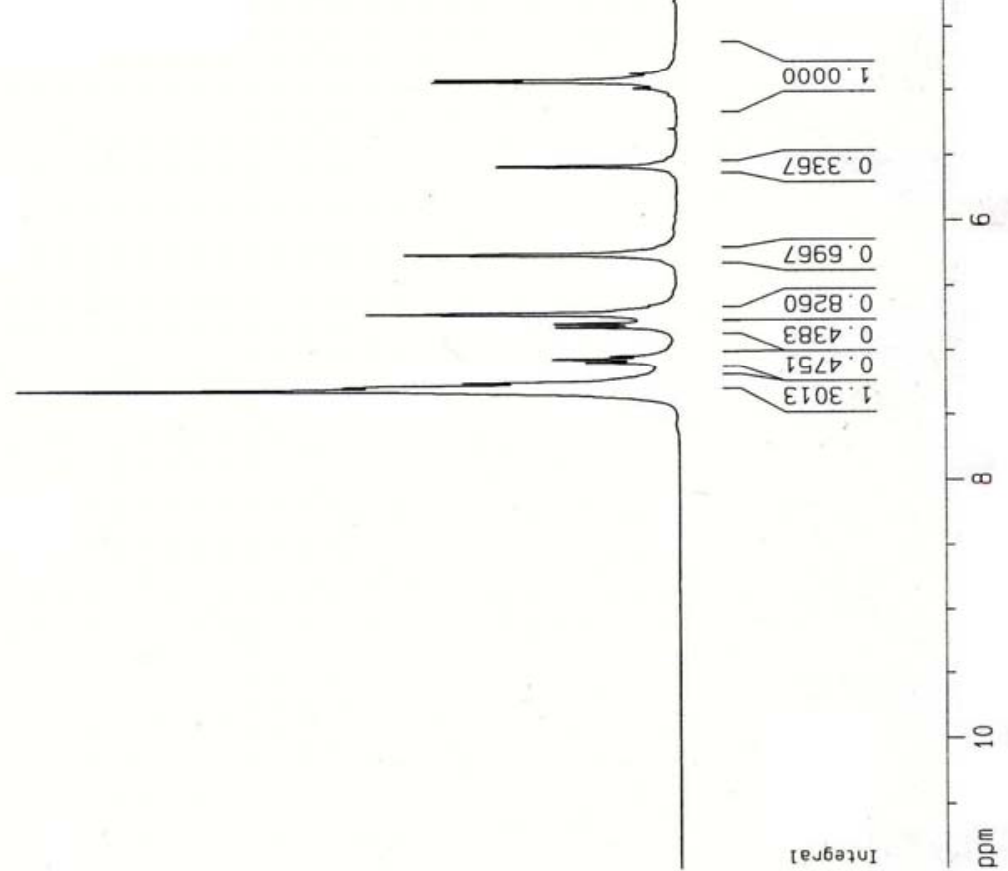




\section{S11}

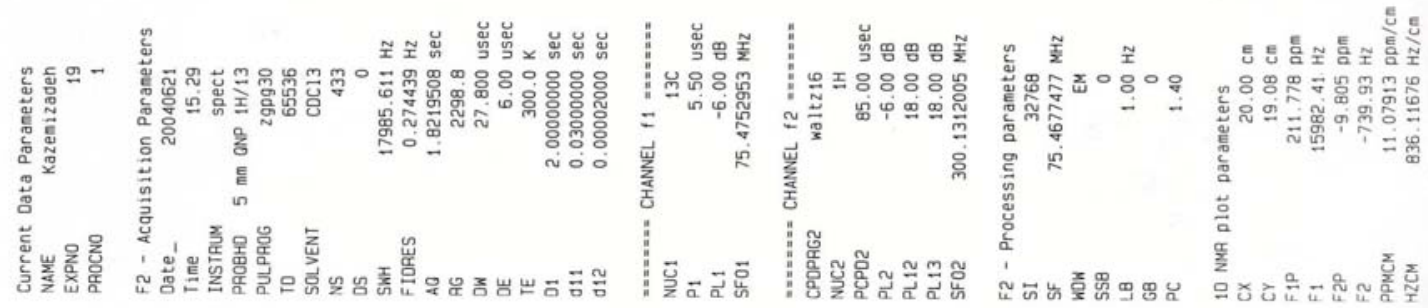

$\varepsilon 90^{\circ} 0$

ICI' $\Rightarrow 0$

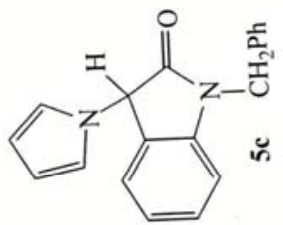

I $\angle 9^{\circ} 9 \angle$

$960^{\circ} \angle L$

86E. 09
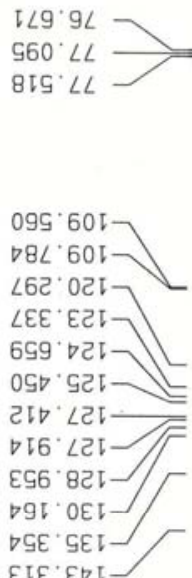

EIE.

$\varepsilon 89^{\circ} \mathrm{LL}$

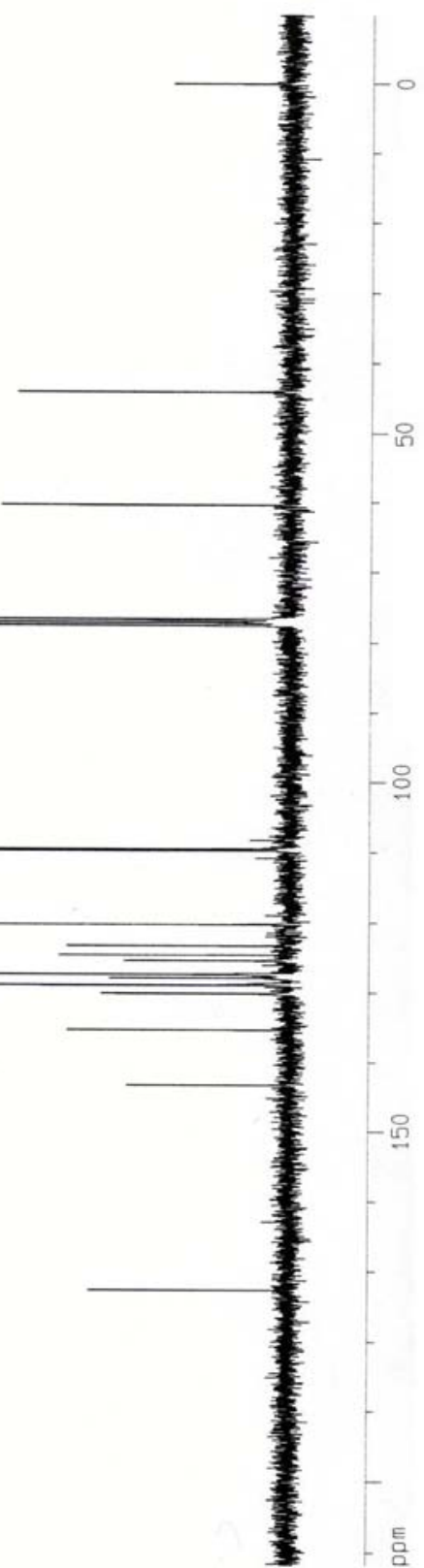




\section{S12}

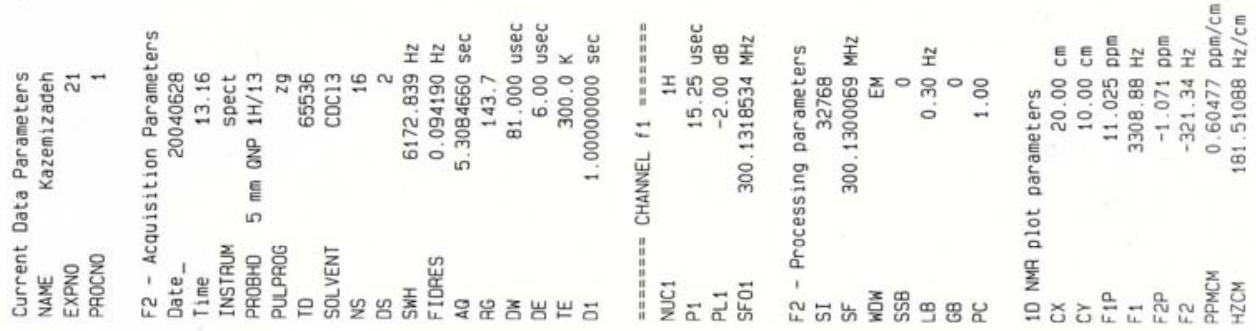

$5000^{\circ} 0-$

至

ट88टं ट

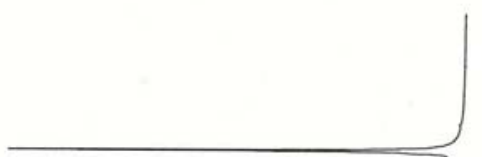

$\bar{a}$

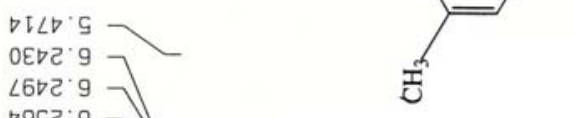

เ

$\checkmark 995$ '

$\checkmark 969 \cdot 9$

$020<\cdot 9$

$580<\cdot 9$

¿ $\angle 6 \angle ' 9$

टЕट८: 9

$\varepsilon 0 \angle 0^{\circ} \angle$

$5080^{\circ} \mathrm{L}$

8टा1: $L$

$\varepsilon 060^{\circ} 6$

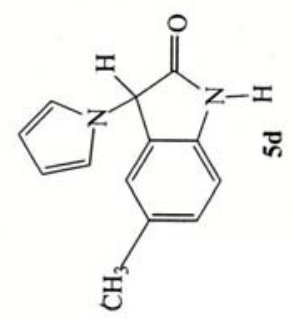

$600<2$

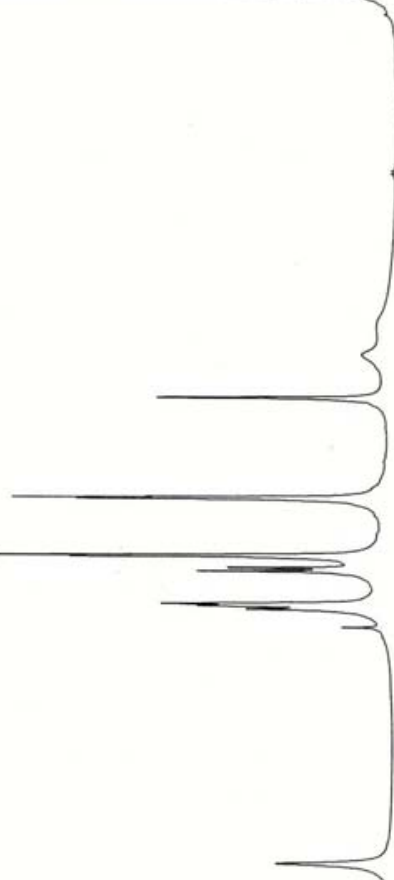

$9668^{\circ} 0$

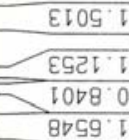

$8059^{\circ} \mathrm{T}$

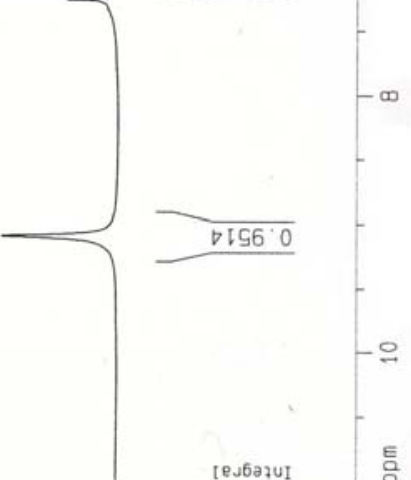

wdd

โอง6aวuI 


\section{S13}

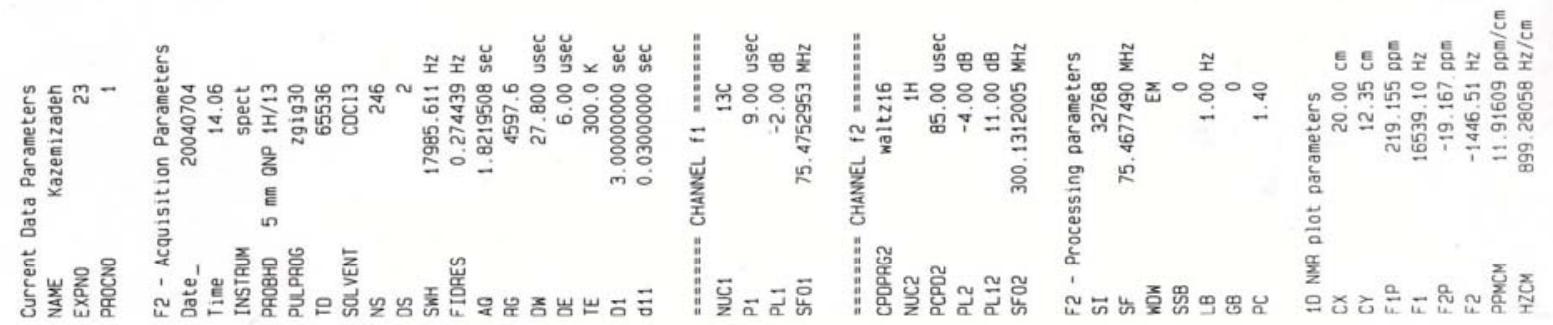
$020 \%$

620.12
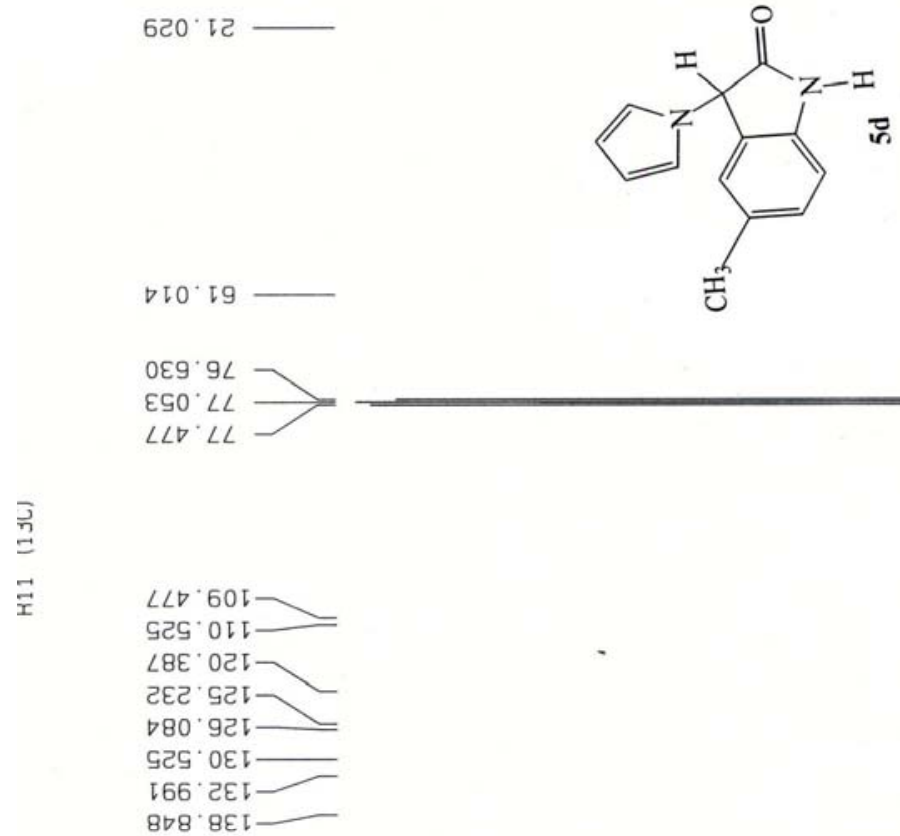

oहg. $9 L$

$\angle \angle D^{\circ} \angle L$

$8 \nabla 8 \cdot 8 \varepsilon 1-$

$\angle O E^{\prime} G \angle I$

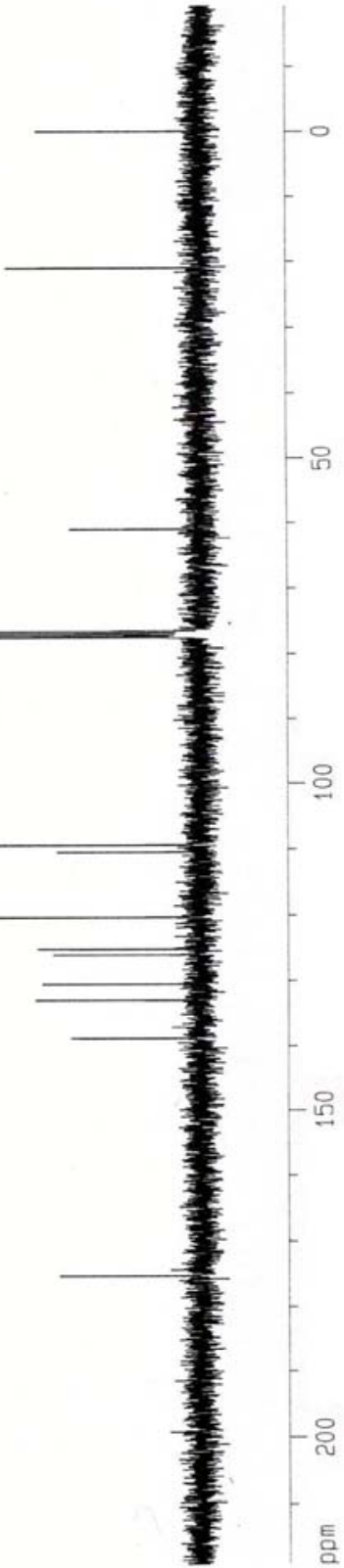

